\section{Custos hospitalares da meningite causada por Streptococcus pneumoniae na cidade de São José dos Campos, São Paulo, Brasil}

\author{
Hospital costs related to streptococcal meningitis \\ among children in São José dos Campos, \\ São Paulo State, Brazil
}

\author{
1 Faculdade de Medicina, \\ Universidade de São Paulo, \\ São Paulo, Brasil. \\ 2 Hospital Universitário de \\ Taubaté, Universidade de \\ Taubaté, Taubaté, Brasil. \\ Correspondência \\ B. R. Lucarevschi \\ Instituto da Criança, \\ Faculdade de Medicina, \\ Universidade de São Paulo. \\ Av. Itália 1551, R6 426, \\ Taubaté, SP 12030-212, Brasil. \\ bfh@usp.br
}

\begin{abstract}
Knowledge of hospital costs is highly important for public health decision-making. This study aimed to estimate direct hospital costs related to pneumococcal meningitis in children 13 years or younger in the city of São José dos Campos, São Paulo State, Brazil, from January 1999 to December 2008. Data were obtained from medical records. Hospital costs were calculated according to the mixed method for measurement of quantities of items with identified costs and value attribution to items consumed (micro-costing and gross-costing). All costs were calculated according to monetary values for November 2009 and in Brazilian currency (Real). Epi Info 3.5.1 was used for frequencies and means analysis. Fortyone cases were reported. Direct hospital costs varied from $R \$ 1,277.90$ to $R \$ 19,887.56$ (mean $=R \$$ 5,666.43), or 10 to 20 times the mean cost of hospitalization for other diseases. Hospital staff labor was the highest cost, followed by medication, procedures, supplies, and lab tests.
\end{abstract}

Pneumococcal Meningitis; Health Care Costs; Costs and Cost Analysis
Bianca Rezende Lucarevschi 1,2 Ana Maria de Ulhôa Escobar 1 Sandra Grisi 1

O pneumococo é reconhecido como um importante agente causal de morbidade e mortalidade em indivíduos de todas as idades há mais de 100 anos. As crianças frequentemente desenvolvem doença pneumocócica do trato respiratório e, menos frequentemente, sepse e meningite. Apesar de haver mais de um século de pesquisas na área, muitos aspectos da doença pneumocócica continuam obscuros. A continuidade da sua ocorrência e severidade, associadas ao conhecimento de que a terapia antimicrobiana, muitas vezes, não cura a doença e, algumas vezes, nem mesmo evita a morte, e a emergência de cepas resistentes aos principais antimicrobianos mostram a real e atual necessidade de conhecer melhor as infecções pneumocócicas 1,2.

Antes do surgimento dos antibióticos e quimioterápicos, a meningite pneumocócica invariavelmente apresentava um desfecho fatal. Com o surgimento das sulfonamidas, na década de 1930, e da penicilina, na década de 1940, a meningite pneumocócica passou a ser uma doença curável. No entanto, apesar da eficácia e efetividade dos antibióticos, cada vez mais potentes, e dos contínuos avanços da terapia intensiva, a morbidade e mortalidade relacionadas à meningite pneumocócica não têm mudado de maneira significativa há décadas, mantendo-se sempre muito altas 3 .

Como programas de vacinação significam investimentos presentes para retornos futuros, o conhecimento dos custos das doenças imuno- 
preveníveis, em especial os custos hospitalares da meningite pneumocócica, é de grande importância para os processos de tomada de decisão no que se refere a intervenções ou estratégias de saúde pública. Gestores e financiadores de procedimentos hospitalares poderão compreender melhor a questão econômica relacionada à meningite pneumocócica, o conceito de custo, os métodos e fontes adotados e sua relação com os resultados das avaliações econômicas, como, por exemplo, avaliações de custo-efetividade de estratégias de vacinação.

O objetivo foi estimar os custos hospitalares diretos relacionados à meningite pelo Streptococcus pneumoniae em crianças com idade até 13 anos (inclusive), na cidade de São José dos Campos, São Paulo, Brasil, em um período de dez anos.

\section{Métodos}

Estudo retrospectivo de custo de doença a partir dos casos notificados de meningite bacteriana aguda, causada pelo S. pneumoniae (meningite pneumocócica), ocorridos de janeiro de 1999 a dezembro de 2008. Critérios de inclusão: casos de meningite bacteriana aguda causada por S. pneumoniae, confirmados e notificados ao centro de vigilância epidemiológica municipal, ocorridos no período do estudo, acometendo crianças até 13 anos (inclusive) e residentes na cidade de São José dos Campos. Os dados obtidos a partir das fichas de notificação compulsória ao Serviço de Vigilância Epidemiológica (Sistema de Informações de Agravos de Notificação - SINAN) mostraram que os casos de meningite pneumocócica ocorridos no período de estudo foram, invariavelmente, tratados no Hospital Municipal Dr. José de Carvalho Florence, que é o hospital de referência para casos de alta complexidade no município e, particularmente, para os casos de meningite bacteriana.

O cálculo da estimativa de custos hospitalares foi realizado de acordo com o método misto para a mensuração das quantidades dos itens de custos identificados e também para atribuição de valor aos itens consumidos, fazendo uso do micro-costing, quando esse era possível, e do gross-costing, como alternativa de viabilidade. $\mathrm{O}$ micro-costing é um método de custeio que reflete as características da amostra (pacientes, instituições), com um alto grau de detalhamento. No entanto, nem sempre é possível utilizá-lo, pois nem sempre os dados estão disponíveis. Por sua vez, o gross-costing reflete custos correspondentes a componentes agregados, abrangendo características da própria instituição estudada e da região em que se encontra, apresentando, assim, importante validade externa. O método misto, utilizado neste estudo, combina os dois métodos, de acordo com a disponibilidade dos dados, e é o adotado pelo Manual de Custeio do National Health Service (NHS) do Reino Unido, bem como pelo setor de faturamento do hospital em estudo $4,5,6,7$. Os dados referentes a esses itens foram obtidos dos prontuários médicos.

O valor dos custos unitários de medicações e materiais utilizados foi atribuído utilizando-se o método do micro-costing, com base no Banco de Preços em Saúde, versão 1.0.5 (Ministério da Saúde; http://portal2.saude.gov.br/BPS/visao/ consultapublica/publico_interno_item.cfm). Esses preços foram utilizados como representativos dos custos de oportunidade, tendo em vista que são os utilizados pelo setor de faturamento do referido hospital.

Para o cálculo do custo dos procedimentos e das diárias hospitalares, foi utilizado o Sistema de Gerenciamento da Tabela de Procedimentos, Medicamentos, Órteses, Próteses e Materiais Especiais do Sistema Único de Saúde - SIGTAP (Departamento de Informática do SUS. http:// sigtap.datasus.gov.br/tabela-unificada/app/sec/ inicio.jsp, acessado em Nov/2009).

Para o cálculo dos honorários profissionais, foi utilizado o valor de remuneração praticado pela Organização Social de Saúde (OSS), pois, como o Hospital Municipal Dr. José de Carvalho Florence é um hospital municipal com gestão privada (gerido pela OSS), os profissionais que nele trabalham recebem salário da OSS e não do município. Foi considerado o número de horas diárias que cada profissional dedicou ao atendimento de cada paciente, durante sua internação pela meningite pneumocócica, com base em registros de atendimento presentes nos prontuários médicos.

Os custos relacionados à energia elétrica, ao abastecimento de água, à rede de esgoto, ao telefone e à manutenção predial são considerados, pelo Hospital Municipal Dr. José de Carvalho Florence, como custos indiretos, e seu valor é determinado por rateio entre os centros de custo. Por isso, não foram incluídos no presente estudo.

Todos os custos foram calculados com os valores monetários referentes a novembro de 2009 e expressos em reais. Para análise das frequências e médias, foi usado o programa Epi Info versão 3.5.1 (Centers for Disease Control and Prevention, Atlanta, Estados Unidos). 


\section{Resultados}

De 1999 a 2008, foram notificados ao núcleo municipal de vigilância epidemiológica 41 casos de meningite pneumocócica em menores com até 13 anos de idade. A média de idade foi de 4,8 anos (mediana $=3,5$ anos; DP $=5,1$ anos), sendo que $56,3 \%$ dos casos ocorreram em crianças de 0 a 2 anos de idade ( $n=23$ ), e $73,3 \%$ dos casos ocorreram em crianças de 0 a 5 anos de idade $(\mathrm{n}=30)$. A maior parte dos casos ocorreu em meninos $(65,6 \% ; n=27)$. A prevalência (número de casos de meningite pneumocócica sobre o número de casos de meningite de todas as etiologias) variou entre $0,48 \%$ e $5,96 \%$ ao longo do período de estudo. Por sua vez, o número de casos variou de 1 a 9 por ano. Pôde-se observar, também, uma maior concentração do número de casos nos meses de inverno (de julho a setembro). O tempo de internação variou entre 8 e 47 dias (média = 23,1 dias; mediana = 19,5 dias; $\mathrm{DP}=14,9$ ). O tempo de permanência na emergência pediátrica variou entre 1 e 6 dias (média = 3 dias). A permanência em unidade de terapia intensiva pediátrica (UTI pediátrica), por sua vez, foi maior, pois variou de 4 a 27 dias (média =7,5). O tempo de permanência em enfermaria de pediatria variou de 6 a 19 dias (média = 13,3 dias).

Dos 41 casos analisados, 10 evoluíram para o óbito (24,4\%; intervalo de $95 \%$ de confiança - IC95\%: 12,4-40,3). Dois pacientes evoluíram com hidrocefalia já durante a internação por meningite aguda $(11,1 \%$; IC95\%: $1,4-34,7)$. No entanto, a complicação mais comumente observada foi a infecção secundária, que ocorreu em 4 casos (22,2\%; IC95\%: 6,4-47,6), sendo um caso de infecção osteoarticular em paciente com anemia falciforme e deficiência de complemento (fração C2), um caso de pericardite purulenta e dois casos de pneumonia associada à ventilação mecânica.

Os custos hospitalares diretos variaram de $\mathrm{R} \$ 1.277,90$ a $\mathrm{R} \$ 19.887,56$, com média de $\mathrm{R} \$$ $5.666,43$. Os custos dos honorários profissionais foram os mais relevantes e variaram de $\mathrm{R} \$ 311,00$ a $\mathrm{R} \$ 3.844,95$ (média $=\mathrm{R} \$ 1.211,30$ ), seguidos pelos custos dos medicamentos (de R\$ 60,14 a $\mathrm{R} \$ 2.602,85$; média $=\mathrm{R} \$ 632,95)$, procedimentos (de $\mathrm{R} \$ 7,04$ a $\mathrm{R} \$ 1.655,24 ;$ média $=\mathrm{R} \$ 846,77$ ), materiais (de R\$15,42 a R\$1.083,08; média $=R \$$ 210,24 ) e exames laboratoriais (de $\mathrm{R} \$ 18,30$ a $\mathrm{R} \$$ 324,67; média = R\$ 161,32) (Figura 1).

Com relação à antibioticoterapia, em apenas um dos casos, foi usada a penicilina (que é o antibiótico de escolha para o tratamento da meningite pneumocócica de acordo com as diretrizes clínicas atualmente vigentes 7), em associação à ceftriaxona. A vancomicina $(\mathrm{R} \$ 5,27 / 500 \mathrm{mg})$ foi amplamente usada, ora como monoterapia, ora em associação à ceftriaxona (R\$ 6,00/1g), à cefepima (R\$14,50/1g) ou até mesmo ao imipenem (R\$ 16,20/500mg), para o tratamento inicial

\section{Figura 1}

Custos hospitalares relacionados a materiais e medicamentos, exames laboratoriais, procedimentos e honorários profissionais, por paciente.

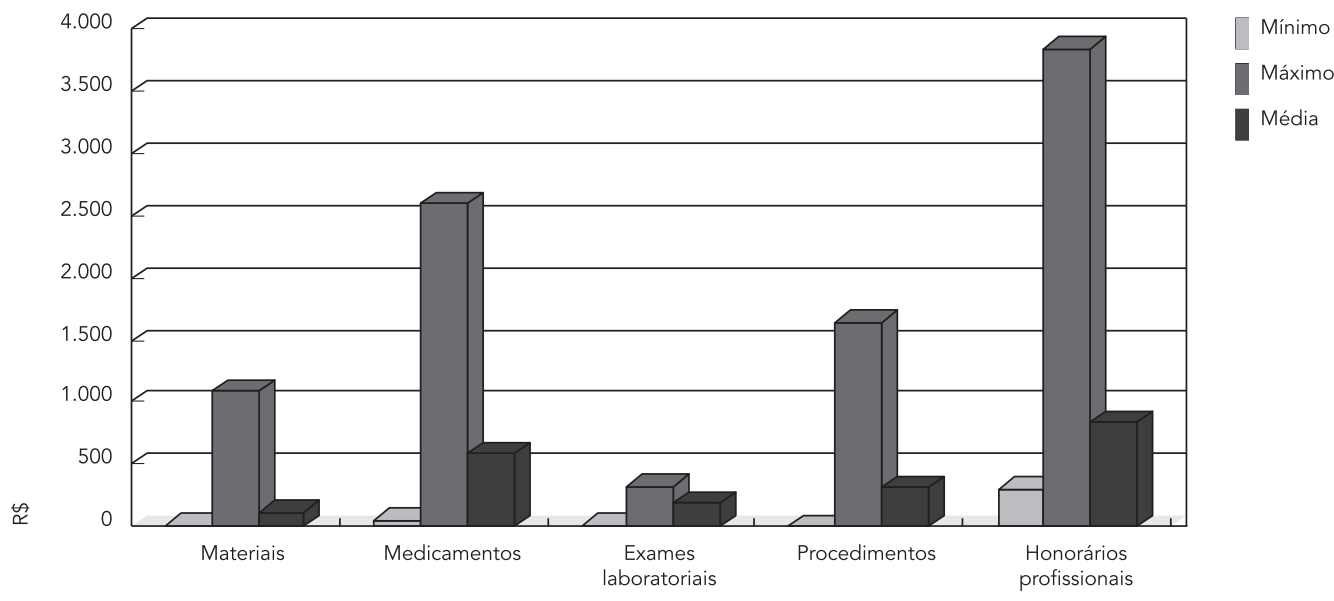


da meningite ou para o tratamento de infecções secundárias.

A UTI pediátrica foi a unidade que apresentou os maiores custos, seguida pela enfermaria de pediatria e, por último, a emergência pediátrica (Tabela 1).

O custo anual total do tratamento hospitalar das meningites pneumocócicas foi muito variável, mesmo considerando a grande diferença do número de casos por ano (Tabela 2).

\section{Discussão}

O presente estudo analisou os custos hospitalares diretos, com base em dados de prontuários médicos, utilizando o Banco de Preços em Saúde. Esse método foi o escolhido por ser o mais frequentemente utilizado em estudos de custo de doença 8,9 e também por ser o recomendado pela Organização Mundial da Saúde (OMS) 10 e pelo próprio Ministério da Saúde 11. Além disso, o estudo foi realizado em um hospital público (municipal), e, no caso de compras de medicamentos feitas pelo governo, pode-se considerar que o Banco de Preços em Saúde reflita os preços praticados em negociações públicas, como licitações e pregões 6 .

A metodologia utilizada apresenta os custos unicamente da perspectiva do sistema de saúde pública e não leva, em conta, a perspectiva da sociedade, em que os custos indiretos também seriam incluídos. Mas o presente estudo apresenta um grande diferencial com relação à maioria dos estudos de custo de doença presentes na literatura: analisou dados de prontuários médicos para verificar itens de consumo e estimar custos, enquanto a maior parte dos estudos (que, por sua vez, também são escassos) considerou modelos de tratamento baseados em diretrizes clínicas para essa estimativa 6,9,12,13 ou mesmo baseados em estudos anteriores 14. Dessa forma, pode-se inferir que os dados aqui apresentados reflitam os custos de maneira muito mais próxima dos

Tabela 1

Custos hospitalares relacionados à unidade de internação hospitalar, por paciente.

\begin{tabular}{lccc}
\hline Custo & Mínimo (R\$) & Máximo (R\$) & Média (R\$) \\
\hline Emergência pediátrica & 0,00 * & $1.498,59$ & 488,02 \\
UTI pediátrica & $0,00 *$ & $17.956,74$ & $3.910,33$ \\
Enfermaria & 0,00 * & $4.030,01$ & $2.084,02$ \\
\hline
\end{tabular}

* Houve casos que não foram admitidos nessas unidades.

Tabela 2

Custo anual do tratamento hospitalar da meningite pneumocócica em São José dos Campos, São Paulo, Brasil, no período de 1999 a 2008 .

\begin{tabular}{|c|c|c|c|c|c|}
\hline \multirow[t]{2}{*}{ Ano } & \multirow[t]{2}{*}{ Número de casos } & \multicolumn{4}{|c|}{ Custo (R\$) } \\
\hline & & $\begin{array}{c}\text { Mínimo por } \\
\text { paciente }\end{array}$ & $\begin{array}{c}\text { Médio por } \\
\text { paciente }\end{array}$ & $\begin{array}{c}\text { Máximo por } \\
\text { paciente }\end{array}$ & Total no ano \\
\hline 1999 & 1 & $4.516,60$ & $4.516,60$ & $4.516,60$ & $4.516,60$ \\
\hline 2000 & 2 & $1.936,23$ & $6.606,92$ & $11.277,61$ & $13.213,84$ \\
\hline 2001 & 9 & $3.519,40$ & $8.067,51$ & $13.419,59$ & $72.607,63$ \\
\hline 2002 & 5 & $1.874,98$ & $3.190,51$ & $3.519,40$ & $15.952,58$ \\
\hline 2003 & 7 & $2.630,76$ & $3.410,24$ & $4.532,58$ & $23.871,70$ \\
\hline 2004 & 3 & $2.630,76$ & $4.948,60$ & $7.682,46$ & $14.845,80$ \\
\hline 2005 & 2 & $1.277,90$ & $1.954,33$ & $2.630,76$ & $3.908,66$ \\
\hline 2006 & 6 & $1.936,23$ & $7.451,49$ & $19.526,82$ & $44.708,99$ \\
\hline 2007 & 2 & $7.080,04$ & $13.483,80$ & $19.887,56$ & $26.967,60$ \\
\hline 2008 & 4 & $2.184,84$ & $5.141,23$ & $6.126,70$ & $20.564,94$ \\
\hline
\end{tabular}


custos reais, embora sejam ainda considerados como estimativas de custos. A estimativa de custos de maneira mais detalhada, como a realizada neste estudo, é considerada de difícil realização, consome muito tempo e pode sofrer grande variação ao longo do tempo, de acordo com a estabilidade econômica do país em que é realizada.

A grande variedade de métodos de estimativa de custos dificulta a generalização das conclusões dos diferentes estudos de custo de doença. Ainda mais: mesmo em estudos de custos de hospitalizações com metodologias semelhantes, o simples fato de serem realizados em hospitais diferentes, em Unidades Federativas diferentes, restringe a possibilidade de generalização de suas conclusões, pois cada estudo reflete as condições da instituição em que foi realizado 15,16,17.

A falta de registro das medicações e dos materiais consumidos foi uma constante nos prontuários estudados. Os materiais médicos e medicamentos exercem um importante impacto nos custos diretos hospitalares. Neste estudo, em particular, que se baseou em dados de prontuários médicos, o impacto desse componente nos custos totais pode ter sido subestimado devido a essa lacuna nos registros. Esse fato pode refletir a falta de preocupação dos profissionais de saúde com os custos hospitalares. De fato, não há nenhuma disciplina relacionada à economia em saúde nas escolas médicas. O tema é desconhecido da maioria dos profissionais de saúde, principalmente em hospitais públicos, o que torna ainda mais difíceis a gestão de custos e as tomadas de decisão.

Quanto aos valores atribuídos aos procedimentos na tabela de referência nacional do SUS, observa-se um evidente subfinanciamento, resultado de uma defasagem com relação aos preços de mercado ocorrida ao longo dos anos. A tabela de procedimentos utilizada como referência neste estudo foi implantada na década de 80, como parte do Sistema de Assistência Médico-Hospitalar da Previdência Social (SAMHPS), posteriormente renomeado como Sistema de Informações Hospitalares (SIH; a partir de 1991). Em 2008, passou a vigorar a nova Tabela de Procedimentos, Medicamentos, Órteses, Próteses e Materiais Especiais do SUS, com o objetivo de torná-la um instrumento de gestão para as ações de planejamento, programação, regulação, avaliação, controle e auditoria em saúde, bem como integrar as bases de informações do Sistema de Informações Ambulatoriais (SIA) e SIH do SUS a fim de construir um sistema unificado. Com a criação dessa nova tabela, houve um reajuste geral dos preços na tentativa de melhorar a situação financeira dos hospitais do SUS. No entanto, o subfinanciamento dos procedimentos não foi solucionado. Apesar dessas discrepâncias, o próprio Ministério da Saúde recomenda a utilização de dados oficiais nos estudos de custo de doença, pois os considera como custos do ponto de vista do SUS, além de ter abrangência nacional 10 .

Os recursos financeiros destinados à saúde são limitados e escassos. Além disso, a área da saúde apresenta uma peculiaridade: a incorporação de novas tecnologias tende a ser cumulativa e não substitutiva, como em outras áreas da economia 10. Esse é considerado um dos principais fatores do aumento dos custos em saúde 18 .

Outra peculiaridade relacionada à saúde é o fato de a demanda por novos serviços ser criada pelo provedor, ou seja, é o profissional de saúde, em particular, o médico, quem determina a demanda por serviços mais caros e sofisticados, e não o paciente. Dessa forma, observa-se um desequilíbrio entre necessidades por cuidados de saúde e demanda por esses serviços 6,19.

Por outro lado, os avanços tecnológicos voltados para a prevenção, em especial as vacinas, têm se mostrado efetivos na redução dos custos em saúde. Os estudos de custo-efetividade têm demonstrado que os investimentos em imunizações são custo-efetivos, especialmente no que diz respeito à doença pneumocócica $13,20,21,22,23,24,25,26$. Como o investimento em vacinas é um investimento presente para um retorno futuro e o custo da introdução de uma nova vacina passa a ser, em um primeiro momento, um custo a mais que se soma ao custo do tratamento da doença a ser prevenida, muitas vezes, não é considerado como custo-efetivo por tomadores de decisão menos atentos. Felizmente, o Brasil tem uma forte tradição em imunizações, com um Programa Nacional de Imunizações (PNI; http://portal.saude.gov.br, acessado em Nov/2009) bem-sucedido, que tem considerado as análises econômicas, principalmente de estudos nacionais, para a introdução de novas vacinas 6 .

Do ponto de vista econômico, a vacinação é considerada uma intervenção capaz de melhorar não somente a sobrevida como a qualidade de vida de uma população, o que leva ao desenvolvimento econômico e consequente redução da pobreza dessa mesma população. Como investimento, pode ser comparada ao investimento em educação, pois interfere positivamente não só com a expectativa de vida, mas também com o rendimento intelectual, com a produtividade, com a capacidade de poupança e, por conseguinte, com os investimentos futuros 6,27,28. As evidências da importância da saúde para a geração de riquezas, além do próprio bem-estar, têm se refletido no crescimento mundial do mercado de vacinas 6,29 . 
O presente estudo demonstrou que os custos diretos do tratamento da meningite pneumocócica têm componentes que, a princípio, não seriam contabilizados com base em protocolos técnicos, pois cada paciente apresenta uma evolução em particular, e as equipes que o assistem têm condutas também particulares. Complicações graves, como hidrocefalia e infecções secundárias, foram responsáveis pela elevação dos custos em alguns casos, enquanto, em outros, os custos foram maiores devido ao elevado preço de antibióticos. Houve casos em que foram necessárias intervenções de alto custo, como derivação ventrículo-peritoneal, drenagem torácica e drenagem óssea, essa última ocorrida em um paciente com anemia falciforme e deficiência da fração C2 do complemento. Pacientes com comorbidades como asma brônquica, por exemplo, necessitaram de tratamento específico para elas, o que também contribuiu para o aumento do custo ${ }^{30}$. O mesmo acontece com o custo da utilização dos hemocomponentes.

A particularidade de evolução e condução de cada caso, aliada à ausência de padronização das condutas (em protocolos técnicos, por exemplo), pode explicar, ao menos em parte, o fato de os custos dos honorários profissionais terem sido os mais relevantes, pois surge a demanda pela intervenção de profissionais que, inicialmente, não estariam envolvidos no atendimento ao paciente com meningite (honorários de especialistas relacionados à execução de procedimentos e cirurgias, por exemplo).

Os custos diretos do tratamento hospitalar da meningite pneumocócica não estão nunca somente a ela relacionados. Cada paciente, em sua individualidade, apresenta uma condição de saúde particular e única, com comorbidades, grau de nutrição, maturidade imunológica e capacidade de recuperação únicas. Todos esses fatores interferem na evolução clínica do paciente e, consequentemente, nos custos da sua hospitalização. Essa é uma das principais limitações encontradas na maior parte dos estudos de custo de doença que utilizam o método de soma de diagnóstico específico, que identifica todos os pacientes com um diagnóstico primário e soma todos os custos para o seu tratamento, sem considerar os custos relacionados a comorbidades, dependendo de como essas são atribuídas ao diagnóstico primário 30 .

Quanto ao manejo técnico dos casos, observa-se que a falta de uniformidade na conduta terapêutica eleva significativamente o custo. Esse fato é notório no que diz respeito à antibioticoterapia. As diretrizes clínicas mundialmente aceitas nos últimos dez anos indicam a benzilpenicilina potássica $($ preço $=\mathrm{R} \$ 1,95 / 5.000 .000 U$ ) como antibiótico de escolha para o tratamento da meningite pneumocócica. Como no momento da escolha do antibiótico a ser utilizado raramente se conhece o agente etiológico da meningite, está tecnicamente indicado o uso de cefalosporina de terceira geração (particularmente a ceftriaxona $\mathrm{R} \$$ 6,00/1g) por apresentar espectro de ação mais amplo 2,7,31,32. No entanto, o que se observou foi o uso de outros antimicrobianos em monoterapia ou associações, o que elevou o custo do tratamento antimicrobiano dessa infecção.

Uma hipótese para o uso frequente de antibióticos de espectro mais amplo é a preocupação com a possibilidade de que a cepa de pneumococo em questão apresente resistência à penicilina. Felizmente, a resistência dos pneumococos a cefalosporinas de terceira geração, no Estado de São Paulo, ainda é suficientemente baixa (cerca de $2,5 \%$ ), o que permite que a ceftriaxona continue sendo utilizada empiricamente 33 .

Procedimentos de alto custo, como tomografias computadorizadas, foram responsáveis pela elevação dos custos do tratamento. Observou-se que, em alguns casos, foram realizadas mais de uma punção lombar, bem como várias radiografias de tórax. Sabe-se que, muitas vezes, essa conduta é necessária, devido à má evolução clínica do paciente. Existe também a hipótese de um primeiro procedimento não ter uma qualidade técnica satisfatória e precisar ser repetido para confirmação diagnóstica, com consequente elevação dos custos.

A falta de padronização nas condutas diagnósticas e terapêuticas, somada às diferenças na gravidade e na evolução clínica entre os casos, faz com que os custos sejam muito variáveis caso a caso e mesmo ano a ano.

Conclui-se, então, que os protocolos técnicos e diretrizes clínicas são importantes para a condução dos casos, considerando não somente o desfecho clínico (cura com ou sem sequelas ou óbito), mas também os custos hospitalares, pois são capazes de uniformizar as condutas médicas, respeitando a individualidade de cada caso. De fato, os protocolos técnicos têm sido exigidos atualmente como parte da documentação necessária para a acreditação de um serviço de saúde. E, cada vez mais, os serviços de saúde e os hospitais, em particular, têm buscado essa acreditação 34 .

O tempo de internação também é um fator relevante no que diz respeito aos custos do tratamento. O SUS repassa aos hospitais, para o tratamento da meningite pneumocócica, o valor de $\mathrm{R} \$ 865,91$ (código de procedimento: 03.03.01.003-7) para uma média de permanência de seis dias, podendo chegar a 12 dias. A permanência do paciente por mais tempo é faturada à 
parte (08.02.01.019-9 - permanência maior), com valor diário de $\mathrm{R} \$ 20,06$. As diárias de UTI também são faturadas à parte (08.02.01.007-5 - R\$ 508,00) (Departamento de Informática do SUS. http://sigtap.datasus.gov.br/tabela-unificada/ app/sec/inicio.jsp, acessado em Nov/2009). De fato, atualmente, o gerenciamento de leitos tem sido uma preocupação dos gestores em saúde. O tempo de internação deve ser o menor possível, desde que respeite as condições clínicas de cada paciente.

Os valores encontrados neste estudo, com relação ao custo médio da internação por paciente, são muito maiores quando comparados com os valores médios pagos por internação hospitalar no SUS (AIH), em pediatria, no Estado de São Paulo, em todos os anos estudados. O valor médio pago pelas AIH variou de $\mathrm{R} \$ 397,21 \mathrm{em}$ 1999 a R\$ 702,00 em 2007 (Departamento de Informática do SUS. http://sigtap.datasus.gov.br/ tabela-unificada/app/sec/inicio.jsp, acessado em Nov/2009). Observa-se que o tratamento da meningite pneumocócica é, sem dúvida, um tratamento de alto custo, chegando a custar 10 a 20 vezes mais do que o valor médio das AIH.

\section{Resumo}

O conhecimento dos custos hospitalares é de grande importância para os processos de tomada de decisão em saúde pública. O objetivo deste estudo foi estimar os custos hospitalares diretos relacionados à meningite pneumocócica em crianças com até 13 anos (inclusive), na cidade de São José dos Campos, São Paulo, Brasil, de janeiro de 1999 a dezembro de 2008. Foram obtidos dados de prontuários médicos. O cálculo foi realizado pelo método misto de mensuração das quantidades dos itens de custos e atribuição de valor aos itens consumidos (micro-costing $e$ gross-costing). Os valores monetários referem-se a novembro de 2009, sendo expressos em reais. A análise das frequências $e$ médias foi realizada pelo programa Epi Info versão 3.5.1. Foram notificados 41 casos. Os custos hospitalares diretos variaram de $R \$ 1.277,90$ a $R \$ 19.887,56$ (média $=R \$ 5.666,43$ ), ou seja, 10 a 20 vezes maiores que o custo médio de internações pago pelo SUS. Os custos dos honorários profissionais foram os mais relevantes, seguidos pelos custos dos medicamentos, procedimentos, materiais e exames laboratoriais.

Meningite Pneumocócica; Custos de Cuidados de Saúde; Custos e Análise de Custo
Dados obtidos do SIGTAP (Departamento de Informática do SUS. http://sigtap.datasus.gov. $\mathrm{br} /$ tabela-unificada/app/sec/inicio.jsp, acessado em Nov/2009) indicam que o custo do tratamento hospitalar da meningite pneumocócica pelo SUS tem variado de cerca de $\mathrm{R} \$ 1.800,00$, para as meningites sem complicações, até cerca de $\mathrm{R} \$ 16.000,00$, para as meningites com complicações neurológicas graves 13. Esses dados são mais próximos dos encontrados neste estudo, o que reforça a sua validade, bem como o alto custo do tratamento dessa doença em nosso país.

\section{Conclusões}

Os custos do tratamento hospitalar da meningite pneumocócica em São José dos Campos são muito variáveis e dependem da conduta médica e das variações dos casos quanto à gravidade e à evolução clínica. Em todos os casos, são considerados altos, cerca de dez a vinte vezes maiores que o custo médio de internações pago pelo SUS por AIH.

\section{Colaboradores}

B. R. Lucarevschi participou da concepção, projeto, análise e interpretação dos dados; redação do artigo e aprovação final da versão a ser publicada. A. M. U. Escobar e S. Grisi colaboraram na concepção, projeto, análise e interpretação dos dados; revisão crítica relevante do conteúdo intelectual e aprovação final da versão a ser publicada.

\section{Agradecimentos}

Agradecemos a toda a equipe do Hospital Universitário de Taubaté, em particular à Dra. Gabriela Ricordi Bazin, e também a toda a equipe do Hospital Municipal José de Carvalho Florence, em especial ao Dr. Danilo Stanzani Jr., por todo o apoio recebido. 


\section{Referências}

1. Austrian R. Pneumococcus: the first one hundred years. Rev Infect Dis 1981; 3:183-9.

2. Andersen CO. Streptococcus pneumoniae meningitis. Dan Med Bull 2007; 54:189-209.

3. Swartz MN. Bacterial meningitis: a view of the past 90 years. N Engl J Med 2004; 351:1826-8.

4. Mogyorosy Z, Smith P. The main methodological issues in costing health care services: a literature review. New York: The University of York; 2005. (CHE Research Paper, 7).

5. Gyldmark M. A review of cost studies of intensive care units: problems with the cost concept. Crit Care Med 1995; 23:964-72.

6. Valentim J. Avaliações econômicas de programas de vacinação: as estimativas de custos em intervenções preventivas [Tese de Doutorado]. São Paulo: Faculdade de Medicina, Universidade de São Paulo; 2009.

7. Curtis L, Netten A. Unit costs of health and social care, 2006. Canterbury: Personal Social Services Research Unit, University of Kent; 2006.

8. Wong EYH, Chou SL, Lamoureux EL, Keeffe JE. Personal costs of visual impairment by different eye diseases and severity of visual loss. Ophthalmic Epidemiol 2008; 15:339-44.

9. Ozelo MC, Villaça PR, Almeida JOSC, Bueno TMF, Miranda PAP, Karamalis M. A cost evaluation of treatment alternatives for mild-to-moderate bleeding episodes in patients with haemophilia and inhibitors in Brazil. Haemophilia 2007; 13:462-9.

10. World Health Organization. WHO guide for standardization of economic evaluations of immunization programmes. Geneva: World Health Organization; 2008.

11. Departamento de Ciência e Tecnologia, Secretaria de Ciência, Tecnologia e Insumos Estratégicos, Ministério da Saúde. Diretrizes metodológicas para estudos de avaliação econômica de tecnologias em saúde para o Ministério da Saúde. Brasília: Ministério da Saúde; 2007.

12. Bigal ME, Rapoport AM, Bordini CA, Tepper SJ, Sheftell FD, Speciali JG. Burden of migraine in Brazil: estimate of cost of migraine to the public health system and an analytical study of the costeffectiveness of a stratified model of care. Headache 2003; 43:742-54.

13. Vespa G, Constenla DO, Pepe C, Safadi MA, Berezin E, Moraes JC, et al. Estimating the cost-effectiveness of pneumococcal conjugate vaccination in Brazil. Rev Panam Salud Pública 2009; 26:518-28.

14. Choi BCK, Pak AWP. A method for comparing and combining cost-of-illness studies: an example from cardiovascular disease. Chronic Dis Can 2002; 23:47-57.

15. Bittencourt ONS. O emprego do método de custeio baseado em atividades - Activity-Based Costing $(\mathrm{ABC})$ - como instrumento e apoio à decisão na área hospitalar [Dissertação de Mestrado]. Porto Alegre: Universidade Federal do Rio Grande do Sul; 1999.

16. Araújo DV, Tavares LR, Veríssimo R, Ferraz MB, Mesquita ET. Custo da insuficiência cardíaca no Sistema Único de Saúde. Arq Bras Cardiol 2005; 84:422-7.
17. Carvalho DMT. Financiamento da assistência médico-hospitalar no Brasil. Ciênc Saúde Coletiva 2007; 12:879-92.

18. Newhouse JP. Medical care costs: how much welfare loss? J Econ Perspect 1992; 6:3-21.

19. Labelle R, Stoddart G, Rice T. A re-examination of the meaning and importance of supplier-induced demand. J Health Econ 1994; 13:369-72.

20. Whitney CG, Farley MM, Hadler J, Harrison LH, Bennett NM, Lynfield R, et al. Decline in invasive pneumococcal disease after the introduction of protein-polysaccharide conjugate vaccine. $\mathrm{N}$ Engl J Med 2003; 348:1737-46.

21. McIntosh ED, Conway P, Willingham J, Lloyd A. The cost burden of paediatric pneumococcal disease in the UK and the potential cost-effectiveness of prevention using 7 -valent pneumococcal conjugate vaccine. Vaccine 2003; 21:2564-72.

22. Talbot TR, Poehling KA, Hartert TV, Arbogast PG, Halasa NB, Mitchel E, et al. Reduction in high rates of antibiotic-nonsusceptible invasive pneumococcal disease in Tennessee after introduction of the pneumococcal conjugate vaccine. Clin Infect Dis 2004; 39:641-8.

23. Wisloff T, Abrahamsen TG, Bergsaker MA, Lovoll O, Moller P, Pedersen MK, et al. Cost-effectiveness of adding 7 -valent pneumococcal conjugate (PCV-7) vaccine to the Norwegian childhood vaccination programme. Vaccine 2006; 24:5690-9.

24. Poehling KA, Talbot TR, Griffin MR, Craig AS, Whitney CG, Zell E, et al. Invasive pneumococcal disease among infants before and after introduction of pneumococcal conjugate vaccine. JAMA 2006; 295:1668-74.

25. Kyaw MH, Lynfield R, Schaffner W, Craig AS, Hadler J, Reingold A, et al. Effect of introduction of the pneumococcal conjugate vaccine on drug-resistant Streptococcus pneumoniae. N Engl J Med 2006; 354:1455-63.

26. Bergman A, Hjelmgren J, Rtqvist ARO, Wisløff T, Kristiansen IS, Gberg LDH, et al. Cost-effectiveness analysis of a universal vaccination programme with the 7 -valent pneumococcal conjugate vaccine (PCV-7) in Sweden. Scand J Infect Dis 2008; 40:721-9.

27. Bloom D, Canning D, Weston M. The value of vaccination. World Economics 2005; 6:15-40.

28. World Health Organization. Vaccine introduction guidelines. Adding a vaccine to a national immunization programme: decision and implementation. Geneva: World Health Organization; 2005.

29. Galambos L. What are the prospects for a new golden era in vaccines? Eurohealth 2008; 14:12-4.

30. Okazaki R. Identificação de ineficiências na cadeia de valor da saúde a partir da análise da gestão de compras de quatro hospitais privados no Município de São Paulo [Tese de Doutorado]. São Paulo: Fundação Getúlio Vargas; 2006.

31. Teele DW. Pneumococcal infections. In: Feign RD, Cherry JD, editors. Textbook of paediatric infectious disease. $6^{\text {th }}$ Ed. Philadelphia: Saunders Elsevier; 2009. p. 1288-341. 
32. Musher DM. Streptococcus pneumoniae. In: Mandell GL, Bennet JE, Dolin R, editors. Principles and practice of infectious diseases. 7th Ed. Philadelphia: Elsevier Inc.; 2010. p. 2623-7.

33. Carvalhanas TRMP, Brandileone MCC, Zanella RC. Meningites bacterianas. Boletim Epidemiológico Paulista 2005; 17:15-26.
34. Organização Nacional de Acreditação. Manual brasileiro de acreditação. Brasília: Organização Nacional de Acreditação; 2006.

Recebido em 29/Jun/2010

Versão final reapresentada em 26/Out/2011 Aprovado em 23/Nov/2011 